\title{
Prevalencia de Streptococcus pneumoniae resistente a penicilina en niños que asisten a jardines infantiles en Santiago
}

\author{
Olivia Trucco A.'; Valeria Prado J.'; Jaime Inostroza S. ${ }^{2}$; Elsa Cabrera J. ${ }^{3}$; \\ Raúl Teran R. ${ }^{3}$; Ignacio Bravo P. ${ }^{7}$; Loriana Castillo D. '; Rosa Bustos V. ${ }^{4}$
}

\section{Penicillin resistant Streptococcus pneumoniae among children attending day care centers}

\begin{abstract}
Pneumococcol infections are serious diseoses in chidren. Epidemiclogical changes in Streptocxcus oneumoniae including increosed i-cidence, eoidemic outbreaks and develcpment and soreading of cntimicrobial res stance heve been recognizec. Corrier studies a'e good to monitore artimicrobial resistance and orevoient serotypes. To desc'be prevalence $o^{\prime}$ resistant isolctes of $S$. pneumonae arrong children oltending doy cae centers and their most prevalent serohyes, samples a nosopharyngeol secretions were obtoined and cultured in sheep biooc cgar with or without gentamisin $5 \mu \mathrm{g} / \mathrm{rl} \mathrm{a}^{+} 37^{\circ} \mathrm{C}$ in $\mathrm{C} 5 \% \mathrm{CO}$. Susceptibiliv est ng wos done by skacillin rest, broth microdilutio- and

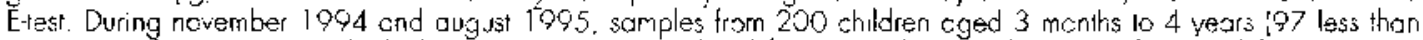
Iwo yeors of agel were studied. S. cneumonige wos isolated from nosopharnx of $63.2 \%$ of these children: $23.4 \%$ isolates were resistart to penicillin (20 3\% moceralely ed uced s Jscoptibi ity ard 8 . '\% Fully resislant strains) and $2 \%$ were resistont to c-loranphenicel No eritromicin, cefolaxime or varcomicin 'es'stont s'rains wero identified. Simiar res stance pctens were opseved among iso oles tom children ethe vounger or older tha cge 2 yegre. Serotype ó was the nost frequently isolcied among diflerent serolypes.
\end{abstract}

(Key words: Streptococcus preiumonice, antibictics, mitrobial cruc resistance. pen icl'irs.l

Las infecciones producidas por Streptoco. ccus pneumoniae representan la principal causa de cnfermedad bacteriana grave en la infancia en todo el mundo, produciendo en los países en vías de desarrollo más de un millón de muertes infantiles por neumonía. También son causa importante de hospitalización y muerte en la tercera edad'.

Streptococcus pnetumoniae es responsable de infecciones sistemicas graves como neumonía, meningitis y septicemia y otras menos severas no invasivas como otitis media aguda, sinusitis aguda, bronquitis y conjuntivitis, en niños y adultos, ubicándose entre los tres agentes más

1. Unidad de Microbiología, Deparımento de Microbinlogía, Facullad de Medicina. Universidad de Chile.

2. Universidad de la Frontera.

3. Ayudantes alumnos, Unidad de Microbiología. Campus Oriente.

4. Tecnó́logo Médico. Instituto de Salud Pública. Finatciamiento: Proyccto Fondecyt N: 1950635. frecuentes ${ }^{2}$. Recientemente este agente ha cobrado importancia por algunos cambios en su epidemiología, entre los que destacan aumento de la incidencia de infecciones neumocócicas en cl mundo, desarrollo creciente de resistencia a penicilina $y$ otros antimicrobianos y resurgimiento de brotes epidémicos en centros de atención cerrada ${ }^{3-5}$.

Estudios de vigilancia en el mundo durante los últimos 5 años han demostrado aumento de I6 a $20 \%$ en la incidencia de infecciones neumocócicas en todas las naciones ${ }^{6}$. La tasa anual de infección, cntre 130 a 210 casos por 100000 habitantes al año, es superior en niños menores de 2 años de edad. ancianos, pacientes con disminución de sus mecanismos de defensa y los infectados con virus de inmunodeficiencia humana $(\mathrm{VIH})^{7}$.

Desde la introducción de la penicilina para ef tratamiento de las infecciones neumocócicas, hace cuatro décadas, este agente se mantuvo sensible a dicho antimicrobiano hasta 1967. en 
que aparecieron las primeras cepas con resistencia moderada a la penicilina (concentración múnima inhibitoria -CMI- 0,1 a $1 \mu \mathrm{gg} / \mathrm{ml}$ ) cn el año 1967 en Australia y Nueva Guinea. Diez años más tarde aparecieron cepas de resistencia clevada (CMI $>2 \mu \mathrm{g} / \mathrm{ml})$ a la penicilina y también a otros antibióticos (multirresistentes). Ningún país se ha mantenido ajeno a la aparición de cepas tesistentes de Streptococcrts phellmoniae, que han ido en aumento, dificultando el tratamiento de los pacientes con estas infecciones ${ }^{3-10}$.

Los estudios de portadores nasofaríngeos de Streptococcus pneumoniae en la comunidad pueden dar una idea de los scrotipos causales de enfermedad invasiva y la resistencia antimicrobiana de estas cepas ${ }^{11}$. Los cambios observados a nivel mundial en las infecciones por Streptococcus preumoniae hacen indispensable estudiarlas también localmente para diseñar esquemas terapéulicos más efectivos.

El proposito de esta investigación fue determinar la frecuencia de colonización nasolaríngea por Streptococcus preumoniae en niños que asisten a salas cuna o jardines infantiles en Santiago; estimar la incidencia de cepas de Streptococcus pnetmoniae resistentes a penicilina $y$ otros antimicrobianos en la flors nasofaríngea; determinar los serotipos de Streptococcus pnetimoniae prevalentes en esos niños y analizar factores de riesgo que favorezcan la colonización por cepas de Streptococciss pheutionice resistentes.

\section{Material y Métodos}

Se estudiaron 200 nin̄os entre 3 mescs y 4 años de edad, de condición sociocconónica media-baja. asistentes a salass cuna o jatdincs infantiles de la Junta Nacional alc Jardines Infantiles (JUNJI).

El estudio de portidores fuc realizado mediante hisopado naxal y faringeo, utilizando torula esteril transportada. al laboratorio de microbiologín del Campus Oriente de la Facullad de Medicina de la Universidad de Chile, en medio de Amics dentro de 6 horas. Las nuestras fueron sembradas paralelamente en plucas de agar sangre (soya tripticasa adicionado de $5 \%$ de sangre de corderob y en placa de agar sangre con agregado de gentamicina $5 \mu \mathrm{g} / \mathrm{ml}$. Las placas fueron incubactos a $37^{\circ} \mathrm{C}$ en aumosfera de 5 a $10 \%$ de $\mathrm{CO}_{2}$ en tarro con vela, por 24 horas.

La identificacion presuntiva de Streplesicas predmontae fue realizada segun normas intemacionalcs ${ }^{12}$. Sc determin 6 la susceptibilidad de las cepas de Streptosocous puesmonite a cinco antimiciobianos (jenicílina. vancomi- cina, eritromicina, cefotaxima, cloranfenicol), empleando un mettodo de rastreo con disco de oxacilina (I $\mu g$ ) para detección de resistencia a penicilina. Sc determins la concentación mínima inhibitoria (CMI) de cada antibiotico mediante lécnica de microdilución en caldo de cultivo. entre \& y $0.0076 \mu \mathrm{g} / \mathrm{m}^{13}$. En e] caso de la penicilina se determinó paralelamente la CMI mediante tiras con gradiente exponencial de pericilina entre 32 y $0,002 \mu \mathrm{g} / \mathrm{ml}$ (E-1est).

Las cepas fucron calificadas de sensibles o resistentes (resistencia intermedia o elevada en el caso de penicilina) según las normas del NCCLS 1994 ${ }^{14}$, 15 . Se utilizó como courral la cepa de $S$, pneumoniae ATCC $496^{10}$.

La serotipificación de las cepas se realizó en el Centro para Control de Enfermedades (CDC) de Atanta, Esiados Unidos. mediante la reacción de Quellung con antisuetos polivalentes y monovalentes.

Los factores de riesgo de colonización por cepas resistentes a penicilina se analizaron en cada niño mediante una encucsta que se completó con ayuda de los padres destinnda a registrar autecedentes de uso de antibióticos en las cuatro semanas precedentes. hospitalización dentro del mes anterior y nivel de hacinamiento.

Los resultados fueron ingresados a uni base de datos tn un programa Windows $2.0 \mathrm{y}$ el analisis estadistico se realizó mediante el programa computacional Epiinfo.

\section{Resultados}

Entre noviembre de 1994 y agosto de 1995 se estudiaron 200 niños de 3 meses a 4 años de edad (97 menores de 2 años y 103 de 2 a 4 años). que asistían regularmente a jardines infantiles o salas cuna de la Junta Nacional de Jardines Infantiles (JUNJI) de Santiago.

En $60,2 \%$ de los niños se aisló Streptococcus pneumoniae de muestras de la nasofaringe. El estudio en paralelo de muestras nasales y faríngeas mostró mejor rendimiento en las primeras, pues todos los portadores albergaban Streptococcus pnetmoniae en la nariz y sólo la mitad de ellos también en la faringe. La frecuencia de aislamicnto de Streptococcus pneumoniae fue similar en menores y mayores de 2 años. El uso de una placa de agar sangre adicionada de gentamicina $5 \mu \mathrm{g} / \mathrm{ml}$ para el cultivo en paralelo de las muestras nasofaríngeas, facilitó el aislamicnto de Streptococus pneumoniae al eliminar una parte importante de flora normal acompañante, facilitando así el manejo de las mucstras. La distribución de serotipos de 50 cepas de $S$. pnetumoniae se muestra en la figura. Al menos 12 serotipos aparecieron diseminados cn la comunidad en secreciones nasofaríngeas, siendo los $6 \mathrm{~A}, 15 \mathrm{~B}$ y $19 \mathrm{~F}$ los más frecuentes. 


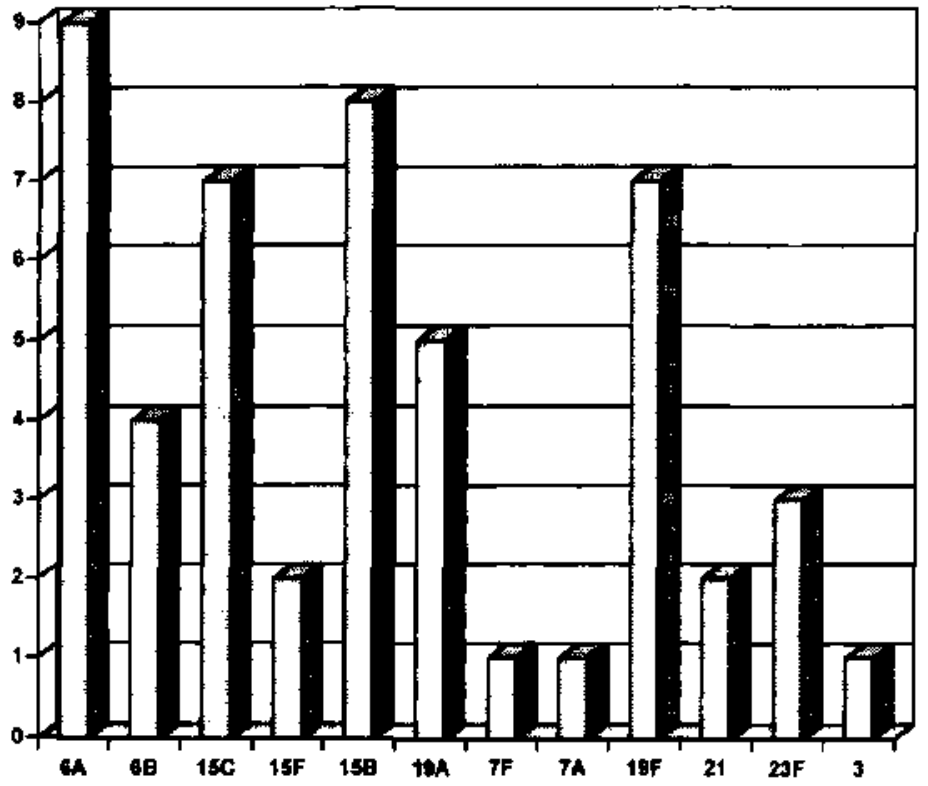

\section{arr serotipos}

Figura: Serotipos de 50 cepas de Streptococors premmunate aisladas de niños portadores nasofaringeos.

En $28,4 \%$ de la cepas se registró algún grado de resistencia a penicilina, $20,3 \%$ presentaban resistencia intermedia (CMI entre 0,1 y $1 \mu \mathrm{g} /$ ml) y $8,1 \%$ resistencia elevada (CMI $>/ 2$ ), todas las cepas eran sensibles a vancomicina, cefotaxima y eritromicina y $2 \%$ eran resistentes a cloranfenicol. El rastreo con disco de oxacilina resultó un buen método para identificar cepas resistentes a penicilina.

El análisis de los posibles fáctores de riesgo de portación de una cepa de Streptococcus pheumoniae resistente a penicilina en la nasofaringe, reveló asociación entre éste y uso previo de antibiótico (el mes anterior), enfermedad respiratoria precedente y mayor grado de hacinamiento familiar (grupo familiar mayor de 5 y 3 o menos habitaciones). En cambio no sc estableció asociación con sexo, edad, hospitalización previa ni enfermedades concomitantes.

\section{Comentario}

Los cambios en la epidemiología de las in. fecciones por Streptococcus pneamoniae han motivado nuevos estudios sobre este patógeno en distintos lugares. Las muestras nasofaríngeas de niños sanos han sido útiles para vigilar su resistencia a los antimicrobianos y predecir los serotipos prevalentes en infecciones invasivas, ya que las cepas obtenides en ambos casos muestran perfiles semejantes de resistencia y distribucion de serotipos ${ }^{16}$, to que permite orientar cn la comunidad las medidas necesarias para el mancjo y prevención de las infccciones producidas por este agente. Chile no ha estado ajeno al problema de la resistencia, ya que en infecciones invasivas se ha encontrado $30,9 \%$ de cepas de resistencia intcrmedia y $4,6 \%$ con resistencia elevada a penicilina ${ }^{17}$.

La diseminación de Streptococcus pneunonise de una persona a otra ha sido demostrada. En jardines infantiles y hospitales para ancianos se han originado pequeños brotes epidémicos de infección neumocócica por una misma ce$\mathrm{pa}^{18,19}$. EI reservorio natural de Streptococcus pneumoniac es la nasofaringe, que se encuentra colonizada entre 5 y $70 \%$ dc los casos, más frecuentemente en niños, especialmente los que asisten a jardines infantiles o salas cuna o viven en centros de atención infantit ${ }^{20,21}$. No está cla- 
ra la relación entre portador nasofaríngeo y enfermedad invasiva, pero sc acepta que ésta sigue al estado de portador (usualmente en la primera semana) de una cepa con capacidad invasiva o de un huesped con deficiencias en los mecanismos de defensa, principalmentc insuficiente actividad de anticuerpos contra el serotipo colonizante ${ }^{2 ?}$.

La frecuencia de colonización nasofaríngea en los riños de este estudio es comparable a muchas series de otros lugares ${ }^{23}$. Se hace notar la gran proporción de estos niños que albergaba al patógeno en la nasofaringe, desde donde es posible su diseminación a territorios cercanes como oído medio, cavidades paranasales, tracto respiratorio inferior o diseminación hematógena, si las condiciones son propicias

En Chilc ha habido un significativo aumento de la resistencia a los antimicrobianos del $S$. pneumoniac. La resistencia a la penicilina en las cepas de porladores nasofaríngeos observada en cste estudio, es comparable a la descrita por otros en cepas aisladas de infecciones invasivas $^{17}$. La resistencia intermedia tiene, al parecer, significación clínica sólo en las infccciones invasivas, $s$ in embargo la resistencia elcvada ha sido asociada a fallas de tratamicnto en olitis media y meningitis, razón por la cual la proporción con que esta última característica ha sido registrada en este estudio sugiere la conveniencia de mantener bajo vigilancia a este patógeno para elegir el tratamiento antimicrobiano más elicaz.

La serotipos más frecuentes en este estudio difieren de los prevalentes en infecciones invasivas. Este cstudio de portadores no es necesariamente un exponente fiel de lo que ocurre con las enfermedades causadas por el agentc en la comunidad pediátrica. En Chile, como en otros países, más de $90 \%$ de los serotipos están incluidos entre los 23 de la vacuna polivalente disponible.

Los factores de riesgo de portar cepas resistentes a penicilina, demostrados en este trabajo, coinciden con lo descrito en otros lugares geográficos, los antimicrobianos betalactámicos facilitan la selección de cepas resistentes y el hacinamiento favorece la diseminación de éstas, por lo que se debe insistir una y orra vez en el uso adecuado y fundamentado de los antimicrobianos en pediatría para retrasar la expansión de cepas resistentes.

\section{Resumen}

Las infecciones neumocócicas causan enlermedades graves en los niños. Sc han observado cambios epidemiologicos en las infecciones por Streptococcus pneamoniae. como aumento de la incidencia, desarrollo creciente de resistencia antimicrobiana y brotes infecciosos epidémicos. EI estudio de portadores es úlil para vigilar la aparición y desarrollo de la resistencia antimicrobiana y de los serotipos prevalentes. Con el propósito de determinar la prevalencia de cepas resistentes de $S$. pnetmonicte en niños que asisten a jardines infantiles de Santiago y los serotipos más frecuentes en la nasofaringe, se efecuó cultivo de hisopado faríngeo y nasal obtenido cntre noviembre de 1994 y agosto de 1995 de 200 de ellos, cuya edad era entre 3 meses y 4 años $(97<$ de 2 años y $103>$ de 2 años). Las muestras fueron sembradas en agar sangre con $5 \%$ de sangre de cordero, con o sin gentamicina ( $5 \mu \mathrm{g} / \mathrm{ml}$ ) e incubadas en ambiente con dióxido de carbono. La resistencia antinnicrobiana se evatuó mediante disco de oxacilina, microdilución en caldo y E-test. En $60,2 \%$ de los casos se aisló S. pnetumoniae de la nasofaringe; $28,4 \%$ de las cepas eran resistentes a penicilina $(20,3 \%$ con resistencia moderada y $8,1 \%$ elevada), $2 \%$ eran resistentes a cloranfenicol. No se encontraron cepas resistentes a eritromicina, cefotaxima o vancomicina. La frecuencia de cepas resistentes a la penicilina fue similar en los niños menores y mayores de 2 años. E serotipo más frecucnte fuc el 6A.

(Palabras clave: Streptococcus pheunoniae, antibiúticos, resistencia microbiana a drogas, penicilinas.)

\section{Referencias}

1. Willians W, Hickson M. Karte M, Kendal A. Spika $I$. Hinmen $A$ : Irmmization policies and vaccine coverage anong adults, Ann Int Med 1988: 108: 616-625.

2. Musher $D$; Sreptorotcur phewnomine. En: Principles and Prictice of Infectious Diseases. Fourth Edition. Editado por Manded, Bennett $y$ Dolin. Churchild Livingstone, New York 1995; 18I1-1826.

3. Butz E. Rodriguez-Creixents M. Muño P. Cercenado $E$. Letmi $M$ : Increased inolation of Streptreceus phetramiate in recent years. Abstr. 1 J76. Progran and Abstracts XXXIII Interscience Conference On Astimicrohial Agents and Chemoherapy. New Odenns. 1993. 
4. Breiman RF, Burter C: Tenore JA. Ellior $J A$ and Facklum RR: Emergence of drug resistant pneumococcal infections in the United States. J Am Med 1994: 271: $1831-18.35$.

5. Cherian $T$, Steinhoff $M C$. Harrism LH. Rein D. $M c D$ ougel $L K$. and Dick $J$ : A cluster of pneumococeal disease in young children in child care. J Am Med 1994: 271: 695-697

6. Breiman R: Changing epidemiology of pnetunocoecul Jisease: a critical necd for vaccine development and use. Symposium: Prevention and Control of preurnococcal infections. XXXIJI Interscience Conference on Antimicrobial Agents and Chemotherapy. New Orlcans 1993.

7. Bouza E. Mafluz P: Penicillin resistaut pneumococci in adult disease with special reference to AJDS patients. Microbial Drug Resistance 1995; 1: 5-28.

8. Hansman D, and Bullen MM: A resistant pueunococcus. Lancet 1967; 2: 264-265.

9. Jecobs MR. Koomhof HJ. Robins-Bron'te RM. et al: Enicrgence of multiply-resistant pneumococci. N Engl J Med 1978; 299: 735-740.

10. Spika Js, Focklom RR, Plikaytis BD. Oxtoby MJ and the Pnewmocecal Surcillence Working Grotg: Antimicrobial resistance of Streptocectur phemomise in the Linited States, 1979-1987. J Inlect Dis 1991: [63: 1273-1278

I1. Musher DM: Infections caused by Strophocectus phenmoniat: Clinical spectrum, pathogenesis. immuniry. and treatment. Clin Infecl Dis 1992; J4: $801-809$.

12. Manual de Lingnóstico bacteriológico en el Lahorarorio Clinico. Instituto de Sulud Pública. 1994.

13. Ruoff K: Streprococtss, En: Manual of Clumical Microbiology, Sixth edition. Editado por Muray. Baron. Pfilles. Tenover y Yolken. American Society for Microbiology, Washingın DC. 1995: 299-307.

14. Performance Siandard for Antimicrobial Disk Susceptibility Tests-Fifth Edition: Approved Slandard. NCCLS Documento M2-A.5.13 N 24, 199?,
15. Method for dilution antimicrobial susceptibility test for bacteria that Grow Aerobically - Third Edition: Approved Standard. NCCLS Documento M7-A.3.13 $\mathrm{N}^{\circ} 25,1993$

16. Mastro D. Nomani $N$. Ghafoor $Z$, et al: Use of nasophatingeal isolates of Streptocectu pneumomiate and Haemophilus influeitarte from children in Pakistan for surveillance of antitnicrobial resistance. Pediatr Infect Dis J 1993; 12: 824-830.

17. Castillo L, Bustos $R$, Ulloa MT, et at: Resistencia antimicrobiana de Streptococus prewmonine causante de infecciones sistémicas en Chile. Revista Chilena dc Infectología 1994: 11: 163-167.

18. Reichler $M R$, Affphin AA. Breiman $K F$. ct at: The sprend of multiply resistant Streprecoccus pneumoniat at a day case center in Ohio. J Infecl Dis 1992: 166: 1.346-1353.

19. Miller MR, Brown NM. Tobin GW, atd Murphy PJ. Oulbreak of infection with penicillin resistant Sirep tococus pnemmonice in a hospital for the eldcriy. $\mathrm{J}$ Hosp Infect 1994: 27:99-104.

20. Masfo TD. Nomani NK. Ishtit $Z$, et at: Use of nasopharyngeal isolates of Streptococtis prewsonitie and Hanophilus inftuenale from children in Pakistan for surveillance for antimicrobial resistance Pediatr Infect Dis 1993: [2: 824-830,

21. Mosedory MC. Camou T, Fajardo $C$, and Hortal M: Colonizing and invasive strains of Streptececcus pesumonime in L'ruguayan children: iype distribution and patterns of antibiotic resistance. Pediatr Infect Dis 199?: 11:648-652.

22. Snth $T$, Lehmenn D. Meutgomery J. Gratten M, Riley ID. and Alpess MP: Acquisition and invasiveness of different serotypes of Sireptococcus preumonias in young children. Epideniol infect 1993; 111: 27-39.

23. Kuernitof H. Warts A, Klugusen $K$ : Antimicrobial resistance in Sereptococets pucumococcol: A South African perspective. Clin Infect Dis. 1992: 15: 84.94. 